\title{
Exploring end of life decision making with people with mild dementia
}

Nathan Davies ${ }^{1,2}$, Tanisha De Souza ${ }^{1}$, Greta Rait ${ }^{1}$, Elizabeth L Sampson ${ }^{2,3}$,

${ }^{1}$ Centre for Ageing Population Studies, Research Department of Primary Care and Population Health, UCL, UK; ${ }^{2}$ Centre for Dementia Palliative Care Research, Marie Palliative Care Research Department, UCL, UK; ${ }^{3}$ Barnet Enfield and Haringey Mental Health Trust Liaison Team, North Middlesex University Hospital ,UK

- Despite efforts to increase advance care planning with people with dementia, many people reach the end of life without one.

- This means there is a lack of clarity around what care the individual would like in certain situations when they are no longer able to express their wishes or making decisions for themselves.

- These decisions may include medical decisions such as the use of artificial nutrition and hydration, on-going treatment of infections, or more social based decisions such as a move to a care home.

- It is often assumed by professionals that families will simply know what the individual would have wanted, and professionals rely on families to make decisions as proxy for the person with dementia.

Family caregivers can feel unsure about decisions and unsupported, leading to feelings of guilt and burden.

- It is important to try and understand from people with dementia themselves how they would like decisions to be made about their care.

There is a lack of work exploring the views of people with dementia about end of life, and what factors they would like their family to consider when making decisions about their care.

\section{Aim}

The aim of this study was to explore with people with mild dementia how they would like family caregivers to make decisions on their behalf at the end of life, exploring factors influencing decisions and what decisions they could make.

\section{Methods}

Design

Qualitative methodology using semi-structured interviews, analysed using thematic analysis methods.

Participants

People with mild dementia were purposively sampled across NHS memory services, genera practice, and research/ third sector networks (e.g. Join Dementia Research, Alzheimer's Society).

Inclusion / exclusion criteria

- Clinical diagnosis of any type of dementia as categorised in ICD-10

- Considered clinically to be in the mild stages dementia

- Mental capacity to provide informed consent

- Ability to read and speak English

- Where there were clinical or social concerns that preclude them being approached people were not eligible

\section{Procedure}

Semi-structured interviews were conducted guided by a topic guide developed with input from people with dementia themselves and family caregivers. Vignettes were used to enable participants to consider situations which they had not encountered yet, and allow the introduction of difficult topics, desensitising difficult aspects through distancing participants from discussing their personal situation to discussing as a vignette character. Interviews were analysed by a team of researchers including two psychologists, a GP, a psychiatrist and input from family caregivers.

\section{Results}

Thirteen interviews were conducted with people with mild dementia in and around the Greater London area, UK.

Participants ranged in age from $72-90$ years and identified predominantly as white British.

A mixture of dementia types was reported including Alzheimer's disease, frontotemporal dementia, and dementia with Parkinson's disease.

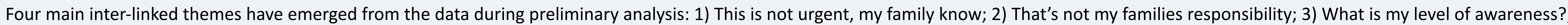
4) Assessing the impact on the family.

\section{This is not urgent, my family know..}

For many they had only discussed with their closest relatives and friends about thei diagnosis. This was a big step in itself and they had not been able to discuss end of life yet. For some end of life was too sensitive, often masked by feelings that these conversation were not urgent.

Many did not consider a need to think about the future and their care. They had faith and trust in their family to make the right decision when the time came. Therefore many participants did not want to discuss in depth how they would like their family or friends to consider making decisions and just leave it alone.

I haven't actually yet [talked to family about end of life]. I say I live day-to-day. I'm not speculating about my personal future, you know. (P026)

Daughter: "We haven't really discussed it, have
we?" Person with dementia: "No, this is the first time that it's - we haven't spoke about - because I didn't think that I was about to pop my clogs." (P023)

"I just try not to sort of dwell on it really. I just think I'll take it as it comes. What's the point of worrying about, you know, all those things

die? You know, lust think I'm sure my children will sort it out if they have to" (P037)
That's not my families responsibility Despite many stating their family would know what to do and that their family were best placed to make decisions about their care. There was a large role for professionals in decision making. There appeared to be a dichotomy about the type of decisions.

Families were seen to be responsible for decisions about financial and legal matters, and any changes in care levels for example moving to a care home or changing care routines at home (i.e. introducing care workers).

Professionals were seen as responsible in discussions with families about medical care including changes in medication, determining what is the best treatment and deciding when "enough is enough" or it is "time to let go". They felt professionals would lead these decisions consulting with family. It would not be fair to leave decisions to have families.

"I can't ask them [family] to make a decision to let me go, if you know what I mean and let me starve and just give me water or whatever it is they do or wet you whistle" (P026)

"Well I would like them [family] to be consulted [by professionals[ before any medical procedure was to go ahead" (P026)
What is my level of awareness?

Interviews with participants were often consumed by discussions about loosing memory and their levels of awareness of people, environment and situations around them.

Level of awareness was described by many people not only as not recognising those around them or their own home, but also a lack of meaning in their responses and no longer being "mentally alert" or making sense becoming an "embarrassment"".

Level of awareness should not only influence the decision itself, for example whether to move the person to a care home, but also an indication of when caregivers should take over and make decisions on the persons behalf.

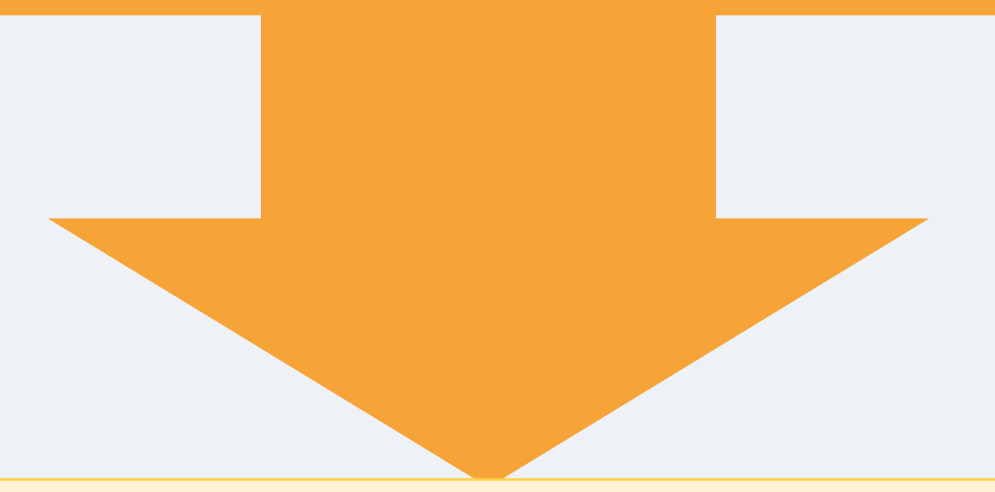

I don't need to go in a care home. No I don't think a care home, oh dear, it all depends on if mentally I'm still alert. (P012)

If I really didn't know what I was doing. It wouldn't matter who would help me go to the toilet, would it? (P036)

"If I was becoming a bit of an embarrassment, I think [family should make decisions]. You know, to myself and to others. [...] I suppose generally, you know, that I couldn't hold a proper conversation but l just talked as my mum did and shouted really." (P037)
Assessing the impact on family

For many participants they were concerned as to what impact their dementia and the care they needed had on the family including the wider family (i.e. grandchildren).

A consideration of the impact on the family was key to many decisions which they felt caregivers may have to make. Participants particularly discussed impact in relation to place of car decisions which often related to the need to give up work, financial constraints, and disruption to daily life generally. Participants were most concerned of being a burden to their family and friends.

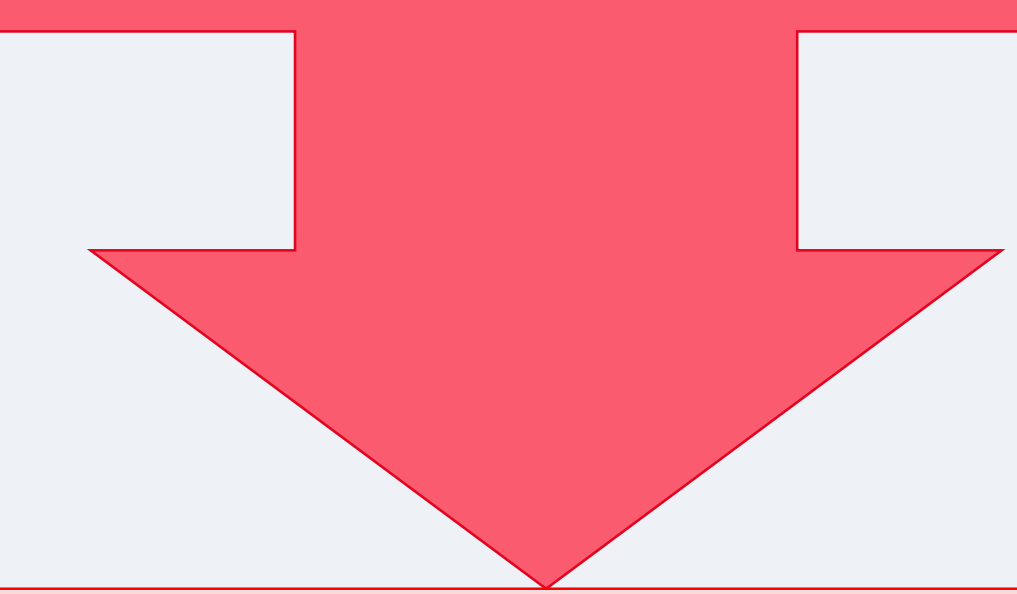

Well, I wouldn't want him [husband] to do everything and be, l'd be a burden to him. I wouldn't want that to happen. Perhaps if he brought in a home help, help me dress and wash. (P036)

If you're incontinent, you don't want to impose that on your family. There are people whose job it is and then I would think, you know, anyone with a job who can stomach it, God help them, lead the job so badly, they have to do it. (P026)

Interviewer: What would you want them [family] to really be considering [about end of life]? Person with dementia: Each other. Not me because I'll not the be there. (P028)

\section{Conclusions}

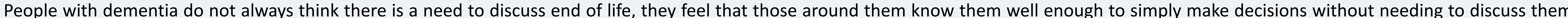

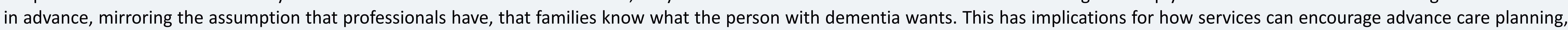
as well as provide services to support families when making decisions.

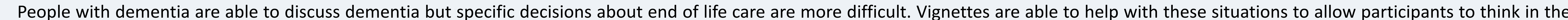
third person and remove some of the ambiguity and emotion of the decision/situation they are confronted with.

- We are continuing to analyse these interviews to inform the development of a decision aid to support family caregivers.

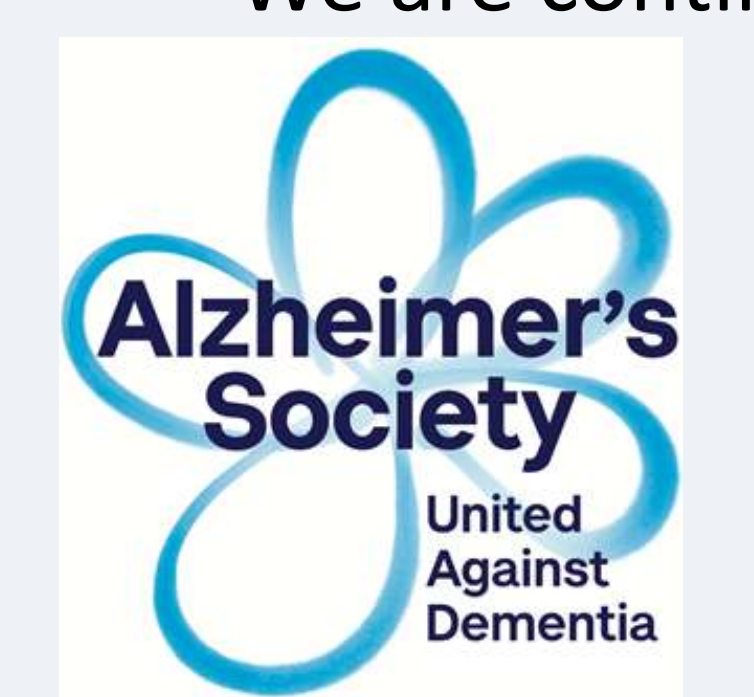

Celebrating 20 years $\quad$ For more details please contact:

Dr Nathan Davies

+44203 1086616

n.m.davies@ucl.ac.uk 